Revue d'histoire de l'Amérique française

\title{
Caritas-Trois-Rivières(1954-1966), ou les difficultés de la charité catholique à l'époque de l'État providence
}

\section{Lucia Ferretti}

Volume 58, numéro 2, automne 2004

URI : https://id.erudit.org/iderudit/011108ar

DOI : https://doi.org/10.7202/011108ar

Aller au sommaire du numéro

Éditeur(s)

Institut d'histoire de l'Amérique française

ISSN

0035-2357 (imprimé)

1492-1383 (numérique)

Découvrir la revue

Citer cet article

Ferretti, L. (2004). Caritas-Trois-Rivières(1954-1966), ou les difficultés de la charité catholique à l'époque de l'État providence. Revue d'histoire de l'Amérique française, 58(2), 187-216. https://doi.org/10.7202/011108ar
Résumé de l'article

À l'origine de la fondation de Caritas-Trois-Rivières, une intention fondamentale : doter le diocèse trifluvien d'un organe de coordination des oeuvres, d'enquêtes sur le terrain, de planification et de financement susceptible d'aider l'Église à conserver l'initiative et la maîtrise d'oeuvre dans le domaine de l'assistance et du bien-être. En dressant l'histoire de cet organisme, nous cherchons dans cet article à mettre en lumière de quelle façon il s'est inscrit dans un milieu en pleine transformation culturelle, et surtout comment il a été affecté par la transition qui s’opère alors au Québec d'une régulation principalement ecclésiastique à une autre quasi exclusivement étatique du système d'assistance et de bien-être. Il appert en effet que si, dans les années 1950, Caritas-Trois-Rivières réussit malgré les difficultés à exercer une activité significative dans le diocèse, la situation change dès le début des années 1960. L'organisme gravite alors de plus en plus dans l'orbite de l'État, jusqu'à en perdre toute raison d'être, en 1966. 


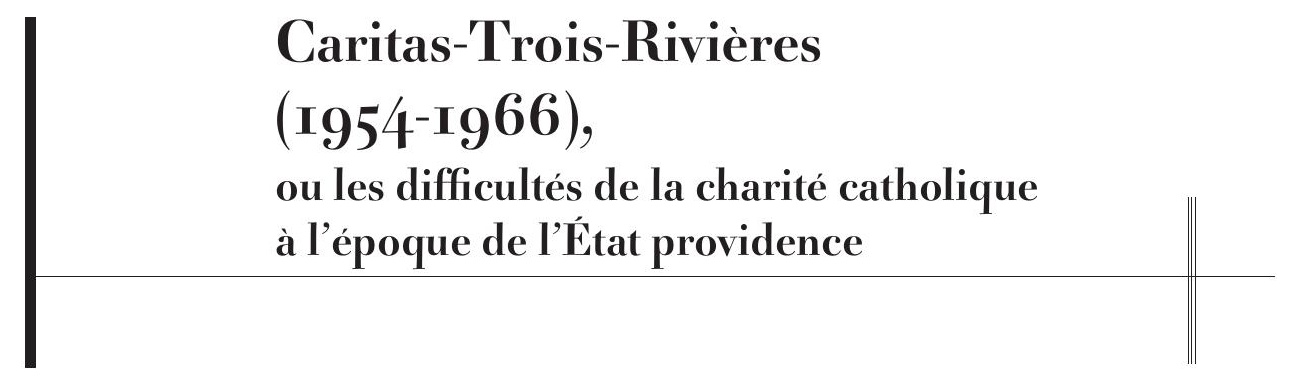

LUCIA FERRETTI

Département des sciences humaines

Centre interuniversitaire d'études québécoises

Université du Québec à Trois-Rivières

\begin{abstract}
RÉsumÉ • À l'origine de la fondation de Caritas-Trois-Rivières, une intention fondamentale: doter le diocèse trifluvien d'un organe de coordination des œuvres, d'enquêtes sur le terrain, de planification et de financement susceptible d'aider l'Église à conserver l'initiative et la maîtrise d'œuvre dans le domaine de l'assistance et du bien-être. En dressant l'histoire de cet organisme, nous cherchons dans cet article à mettre en lumière de quelle façon il s'est inscrit dans un milieu en pleine transformation culturelle, et surtout comment il a été affecté par la transition qui s'opère alors au Québec d'une régulation principalement ecclésiastique à une autre quasi exclusivement étatique du système d'assistance et de bien-être. II appert en effet que si, dans les années 1950, Caritas-Trois-Rivières réussit malgré les difficultés à exercer une activité significative dans le diocèse, la situation change dès le début des années 1960. L'organisme gravite alors de plus en plus dans l'orbite de l'État, jusqu'à en perdre toute raison d'être, en 1966.
\end{abstract}

ABSTRACT - A fundamental purpose lay behind the founding of Caritas-Trois-Rivières, that of providing the diocese of Trois-Rivières with an instrument for coordinating charities, carrying out field investigations, planning and financing that was likely to help the Church maintain the initiative and control in the field of social welfare. In recounting the history of this organization, this article seeks to highlight the manner in which it became part of a community undergoing profound cultural changes and, especially, how it was affected by the transition, in the Québec of the time, from a welfare system governed mainly by the Church to another governed almost exclusively by the State. It would appear, in fact, that while Caritas-Trois-Rivières managed in spite of difficulties to play a highly active role in the diocese 
during the 1950s, this situation changed at the start of the 1960s, when the organization was drawn more and more into the orbit of the State, until by 1966 its existence could no longer be justified.

\section{INTRODUCTION}

Cet article porte sur la naissance, la vie et la mort de Caritas-TroisRivières, qui fut l'organe de coordination, de planification et de soutien financier des œuvres de charité du diocèse de Trois-Rivières entre 1954 et 1966. Nous y serons particulièrement attentive à cerner le projet à l'origine de sa création par l'Église diocésaine, à identifier les ressources sur lesquelles l'organisme a pu compter et qui ont favorisé ses réalisations, certaines durables, mais aussi à repérer les difficultés qu'il a rencontrées et qui ont fini par avoir raison de lui. Nous espérons ainsi pouvoir mettre en lumière avec quels atouts et quelles limites Caritas-Trois-Rivières s'est inscrite dans un milieu en pleine transformation culturelle, puis comment elle a été affectée par la transition qui s'opère alors au Québec d'une régulation principalement ecclésiastique à une autre quasi exclusivement étatique du système d'assistance et de bien-être.

Signe des réorientations qui ont marqué l'existence de Caritas-TroisRivières et des incertitudes entourant les délimitations de ses fonctions, la documentation pertinente pour notre étude est non seulement dispersée dans quatre dépôts d'archives, mais ceux-ci conservent des dossiers qu'on s'attendrait parfois à retrouver ailleurs. À Centraide Mauricie, nous avons pu consulter les cahiers des procès-verbaux des réunions du conseil d'administration et des divers comités du Conseil central des ouvres et de la Fédération des ouvres de charité, noms sous lesquels fut d'abord connue Caritas-Trois-Rivières, puis ceux des organismes qui lui ont succédé, la Fédération des œuvres, le Conseil régional de bien-être de la Mauricie et même, curieusement, la très ecclésiastique Commission diocésaine caritative. Les Archives de l'Évêché de Trois-Rivières conservent plusieurs dossiers relatifs aux campagnes de collecte de fonds annuelles, notamment la correspondance, les nominations, les rapports financiers et les rapports d'activités; ainsi que les statuts et règlements régissant le Conseil central des œuvres, la Fédération et Caritas-Trois-Rivières. Quant au très riche fonds Charles-Édouard-Bourgeois, abrité en partie aux Archives du Séminaire Saint-Joseph et en partie à la procure de l'Évêché, il permet de documenter les liens, qui apparaissent tout à fait intimes, entre le Centre de service social diocésain et Caritas-Trois-Rivières, de 
prendre connaissance de la majeure partie de la couverture de presse régionale concernant les activités de l'organisme et, grâce à l'abondante correspondance qu'entretenait $\mathrm{M}^{\mathrm{gr}}$ Bourgeois avec une foule d'acteurs de la scène du bien-être tant dans l'Église, les milieux professionnels que dans les ministères, de saisir l'ampleur de la transition qui s'est effectuée au cours des années 1960 .

Le cas québécois semble d'ailleurs assez unique à l'échelle occidentale, si on l'examine justement sous l'angle de la rapidité et de la radicalité avec lesquelles s'est faite cette transition. Y compris à l'époque de son apogée, entre les années 1960 et le milieu des années 1980, l'État providence, dans plusieurs pays européens, n'a en effet jamais constitué le tout du dispositif de protection sociale en vigueur. Le secteur privé n'a pas cessé d'y occuper une place significative : par ses entreprises à but lucratif certes, mais aussi par tous les réseaux caritatifs confessionnels, dont plusieurs sont nés au $\mathrm{XIX}^{\mathrm{e}}$ siècle et sont demeurés présents jusqu'à nos jours à côté du secteur public et du marché. Pensons seulement au Secours catholique français ou au Deutscher Caritasverband, auquel Catherine Maurer a consacré une thèse magistrale ${ }^{1}$. Malgré tout, comme le révèle la thèse de Matthias Schmidhalter, l'Église catholique a craint, après le Deuxième Guerre mondiale, d'être marginalisée dans le domaine de l'aide aux populations: c'est pour y garder sa place alors que les États et les organisations publiques internationales telle l'ONU s'y activent désormais, qu'elle a créé Caritas Internationalis, en $1950^{2}$.

Très peu d'études québécoises portent pour leur part sur les conseils d'œuvres, les Caritas diocésaines ou sur les campagnes de collectes de fonds organisées par les Fédérations d'œuvres ${ }^{3}$. Par l'examen du cas

1. Catherine Maurer, Le modèle allemand de la charité. La Caritas de Guillaume II à Hitler (Strasbourg, Presses universitaires de Strasbourg, 1999), 358 p.

2. Matthias Schmidhalter, Caritas Internationalis: Engagée depuis plus d'un demi-siècle pour la solidarité internationale, texte disponible sur le site Internet de Caritas Internationalis, et tiré d'une thèse en préparation à l'Université de Fribourg, en Suisse. http:/ / www.caritas.org/

3. Jules Perron, «L'évolution des services sociaux d'expression française au Québec entre 1940 et 1960 », Intervention, 69 (juillet 1984): 17-27. Louis Beaupré, "Politiques sociales au Québec», Intervention, 69 (juillet 1984): 3-10. «Les années 50 et 60 au Québec: une période de transition pour les pratiques sociales; entrevue avec Louis Beaupré», Nouvelles pratiques sociales, 8,2 (1995): 15-32. (Perron et Beaupré ont été profondément engagés dans les conseils d'œuvres et autres organismes de coordination créés par l’Église québécoise dans les années 1950). Voir aussi: Andrée Désilets et Louise Brunelle-Lavoie, L'Action sociale à Sherbrooke. Caritas-Sherbrooke (1957-1982) (Sherbrooke, Université de Sherbrooke, Département d'histoire, 1982), 75 p. Jacques Saint-Onge, Le développement des pratiques en travail social au Saguenay-Lac-Saint-Jean, 1960-1980 (Chicoutimi, Université du Québec à Chicoutimi, Groupe de recherche et d'intervention régionales, 1999), $252 \mathrm{p}$. 
particulier qu'est Caritas-Trois-Rivières, ce que nous voulons observer, en fait, c'est justement une autre des facettes du processus complexe par lequel le réseau d'assistance et de bien-être diocésain a contribué, sans doute malgré lui mais de manière bien nette, à l'émergence de l'État providence au Québec, pour pratiquement se dissoudre en lui dans les années $1960^{4}$.

\section{I - LE PROJET : PRÉSERVER LA MAîTRISE D'GUVRE DE L'ÉGLISE DANS LE DOMAINE DE L'ASSISTANCE ET DU BIEN-ÊTRE}

Après la Seconde Guerre mondiale, la présence de l’Église catholique dans le domaine de l'assistance et du bien-être au Québec se fait plus multiforme et plus intense que jamais auparavant. Baby boom, prospérité économique, hausse des exigences d'une population marquée par les privations des longues années de crise puis de guerre, financement partiel mais stable qu'apporte la Loi québécoise de l'Assistance publique aux établissements d'hébergement possédés par les congrégations religieuses, implication nouvelle du gouvernement fédéral dans le financement indirect des orphelinats et des instituts de prévention en santé mentale, professionnalisation des services sociaux: tout se conjugue pour encourager l'agrandissement des établissements existants, la création de nouvelles institutions spécialisées, l'ouverture de centres de service social dans tous les diocèses et la multiplication des organismes de charité.

Ce phénomène d'expansion se remarque partout au Québec, et il pousse partout l'Église à la même conclusion: il faudrait une meilleure planification des services sociaux et de la charité à l'échelle diocésaine, une plus étroite coordination des œuvres et trouver aussi le moyen d'assurer à celles-ci un soutien financier plus considérable et plus régulier. En 1947, en tant qu'auxiliaire de l'archevêque, $\mathrm{M}^{\mathrm{gr}}$ Georges-Léon Pelletier travaille donc à doter l'archidiocèse de Québec d'un Conseil central des œuvres, et il met sur pied une campagne diocésaine annuelle de collecte de fonds en leur faveur ${ }^{5}$; avec le vif souci d'éviter que s'y reproduise la situation prévalant à Montréal, où la Fédération des œuvres de charité canadiennes-françaises et le Conseil des œuvres, fondés dans les années 1930, échappent somme toute en grande partie au contrôle de l'archevêque ${ }^{6}$. En 1953, par ailleurs, le prêtre trifluvien

4. Cet article s'inscrit dans un programme de recherche portant sur «Les origines diocésaines de l'État providence : assistance et services sociaux dans le diocèse de Trois-Rivières, 1930-1970", subventionné par le Conseil de recherches en sciences humaines en 2000-2003.

5. Jean Panneton, Le diocèse de Trois-Rivières, 1852-2002 (Sillery, Septentrion, 2002), 120.

6. Lucia Ferretti, «Les agences sociales à Montréal, 1932-1971 ", Études d'histoire religieuse, 66 (2000): 75 . 
Charles-Édouard Bourgeois, ami personnel du Premier ministre Maurice Duplessis et un des promoteurs les plus influents du développement des services sociaux catholiques au Québec, compte parmi ceux qui convainquent les évêques de fonder la Conférence catholique canadienne de bienêtre, qui change de nom pour Caritas-Canada (section française) l'année suivante ${ }^{7}$. Sous l'impulsion de ce nouvel organisme, les diocèses québécois finiront presque tous par s'outiller d'un conseil d'œuvres au cours des années $1950^{8}$.

Quand $\mathrm{M}^{\mathrm{gr}}$ Pelletier est nommé évêque de Trois-Rivières en 1947, il y retrouve l'abbé Bourgeois. Celui-ci dirige l'agence de service social la plus ancienne du Québec français et la mieux structurée ${ }^{9}$. En l'absence d'un conseil d'œuvres, l'Assistance à l'enfant sans soutien exerce même quelques-unes des fonctions qui reviendraient à un tel organisme, notamment une certaine planification des services sociaux aux enfants, la confection d'un bottin des ouvres et la représentation des crèches et orphelinats auprès du gouvernement fédéral et du gouvernement du Québec. C’est Bourgeois qui fait venir les Sours de Miséricorde à Trois-Rivières, en 1944, pour le soin des mères célibataires et de leurs bébés; lui qui obtient de Québec les subsides pour la construction de leur vaste Hôpital SainteMarie, en 1946, ainsi que pour les agrandissements nécessaires aux orphelinats des Dominicaines du Rosaire; et lui, encore, qui propose en 1947 au gouvernement fédéral d'accueillir dans son agence l'Institut psychosocial, consacré au dépistage et au traitement des problèmes mentaux chez

7. J. Perron, loc. cit. Et aussi Archives du Séminaires de Trois-Rivières (à l'avenir ASTR), Fonds 0560, boîte 3, Charles-Édouard-Bourgeois, Rapport de l'Assistance à l'enfant sans soutien pour 1952, 33. Dans cet article, j’abrégerai Caritas-Canada (section française) en Caritas-Canada; à vrai dire, la section anglaise ne fut pas développée avant 1964.

8. A. Désilets et L. Brunelle-Lavoie, op. cit., 6-7.

9. «L'œuvre du Placement de l’orphelin commença en septembre 1931, dès l’entrée de nos pupilles dans le nouvel orphelinat Saint-Dominique, sis boulevard du Carmel. Au printemps de 1934, leur Excellence feu Monseigneur F.-X. Cloutier, évêque de Trois-Rivières, et Monseigneur Alfred-Odilon Comtois, alors évêque auxiliaire, approuvèrent notre Euvre. Quelques semaines plus tard, les Autorités civiles et les diverses associations publiques donnèrent, à leur tour, leur appui officiel", citation tirée du Rapport annuel de l'œuvre de l'Assistance à l'Enfant sans soutien du diocèse des Trois-Rivières, pour 1940, 1, conservé aux Archives de la Ville de Trois-Rivières. Le Placement de l'orphelin est donc l'agence sociale la plus ancienne en milieu canadien-français au Québec. En 1939, l'organisme a changé son nom pour celui de l'Assistance à l'enfant sans soutien, puis de nouveau en 1953 pour celui de Centre de service social du diocèse de Trois-Rivières. Ces changements de nom correspondent à des élargissements successifs dans l'offre de services. Pour un aperçu de l'ampleur du rôle de Charles-Édouard Bourgeois dans le domaine de l'assistance et des services sociaux au Québec, voir: L. Ferretti, "Charles-Édouard Bourgeois, prêtre trifluvien et les origines diocésaines de l'État providence au Québec", Nouvelles pratiques sociales, 14,1 (juin 2001): 169-182. 
les enfants. En fait, parmi les établissements d'assistance de la ville, seuls l'Orphelinat et l'Hospice Saint-Joseph des Sours de la Providence semblent échapper à son attention. L'abbé Bourgeois est aussi depuis 1931 l'orchestrateur de la Journée de la Livre, une campagne de souscription publique qui rapporte plusieurs milliers de dollars chaque année à l'Orphelinat puis à Ville-Joie Saint-Dominique, dont il est l'aumônier. Une partie des surplus ramassés à cette occasion finance d'ailleurs les fonctions de planification, de coordination et de recherche de l'Assistance à l'enfant sans soutien, que le gouvernement ne subventionne en rien ${ }^{10}$. C'est donc en misant sur l'abbé puis monseigneur Charles-Édouard Bourgeois ${ }^{11}$ que $\mathrm{M}^{\mathrm{gr}}$ Pelletier pourra fonder le conseil d'œuvres qu'il souhaite pour son diocèse et mener la campagne annuelle de souscription.

Cela paraît d'autant plus nécessaire qu'à côté de l'impressionnant dispositif institutionnel que nous venons d'évoquer, une myriade de petites œuvres naissent après la guerre : le Gîte reçoit les sans-abri, le Dispensaire Notre-Dame de la Paix soigne les familles très pauvres du quartier du même nom, une Association catholique pour les infirmes assure travail protégé et loisirs à une trentaine d'entre eux, tandis que plusieurs œuvres de la layette et des vestiaires paroissiaux aident les mères à vêtir leurs jeunes. Chacune à sa façon, elles complètent l'action de la très ancienne Société Saint-Vincent-de-Paul ${ }^{12}$. Toutes dépendent du bénévolat, des contributions discrétionnaires des autorités municipales et de la générosité privée. Leur financement, toujours précaire, préoccupe donc quotidiennement leurs dirigeants.

Le projet d'origine se résume en une intention fondamentale: doter le diocèse d'un organe de coordination des œuvres, d'enquêtes sur le terrain,

10. Plusieurs indices ne laissent pas de doute à ce propos: notamment la mention «dons de charité" parmi les rubriques des sources de financement de l'Assistance à l'enfant sans soutien dans les rapports annuels d'activité, alors que l'agence n'a jamais organisé de souscription publique ni privée à son profit; le fait que le premier 1000 \$ reçu par le Conseil central des œuvres, en septembre 1954, provienne «du surplus de la Journée de la Livre de 1953» (Centraide Mauricie, à l'avenir CM), 100A.0159, Cahier des procès-verbaux des réunions $d u$ conseil d'administration du Conseil central des auvres de Trois-Rivières, 15 septembre 1953); et le fait qu'une partie significative des sommes recueillies lors des campagnes de la Fédération des œuvres, à partir de 1954, soit allée au soutien du Centre de service social.

11. L'abbé Bourgeois a été élevé à la dignité de Prélat de la maison du pape en 1952. À partir de cette date, on fait donc précéder son nom du titre de monseigneur: Georges Panneton et Antonio Magnan sr, Le diocèse de Trois-Rivières, 1962 (Trois-Rivières, les Éditions du Bien Public, 1962), 26-28.

12. L. Ferretti et Karl-Xavier Thomas: «Montée de l’État providence et transformations du soutien laïc aux ouvres d'assistance catholiques: Trois-Rivières, 1921-1965 ", Annales de Bretagne et des Pays de l'Ouest, 110,4 (2003): 171-184. 
de planification et de financement susceptible d'aider l'Église à conserver l'initiative et la maîtrise d'œuvre dans le domaine de l'assistance et du bien-être. Certes, sous les gouvernements successifs de Maurice Duplessis, entre 1944 et 1959, l'État est encore loin de représenter une menace pour l'Église dans la régulation de ce secteur. Les indices convergent qui incitent néanmoins le clergé à la vigilance : on craint de devoir bientôt rendre des comptes en contrepartie de l'importante augmentation de la part provenant des fonds publics dans les budgets des établissements privés et, parallèlement, on remarque que l'État finance de plus en plus difficilement l'entrée de l'Église dans de nouveaux secteurs d'activité, la formation professionnelle par exemple ${ }^{13}$. Par ailleurs, le contexte local encourage aussi $\mathrm{M}^{\text {gr }}$ Pelletier à agir: la Chambre de commerce envisage en effet un instant de participer elle-même à la création d'une Fédération des œuvres, non confessionnelle celle-là, pour éviter à ses membres de subir plus longtemps les sollicitations répétées de tous ceux qui quêtent pour les bonnes causes ${ }^{14}$; seul l'ascendant de $\mathrm{M}^{\mathrm{gr}}$ Bourgeois, venu rencontrer ses dirigeants, et son annonce d'une action prochaine de l'évêque réussissent à dissuader les hommes d'affaires. Aussi, la défaite du maire libéral JosephAlfred Mongrain, en novembre 1953, marque-t-elle le moment de passer à l'action: depuis son élection en 1949, il avait cherché à renforcer la capacité de la Ville de Trois-Rivières à agir directement dans l'assistance, plutôt que de subventionner les œuvres d'Église ${ }^{15}$. Son remplacement par le notaire Léo Leblanc, ex-président du Club Richelieu, très proche de l'Église, et administrateur de la Caisse populaire de Trois-Rivières, où l'évêché est client, laisse présager une meilleure réception du projet clérical par le conseil municipal.

En septembre 1954, usant de ses pouvoirs, la Corporation de l'évêque catholique romain de Trois-Rivières édicte deux règlements, par lesquels elle se dote de deux comités. Le Conseil central des ouvres de TroisRivières se voit chargé de promouvoir la coordination des ouvres de charité du diocèse et de procurer à celles-ci aide intellectuelle et technique; tandis que l'organisation d'une campagne annuelle unifiée de

13. L. Ferretti, «L'Église, l’État et la formation professionnelle des adolescents sans soutien: le Patronage Saint-Charles de Trois-Rivières, 1937-1970 ", Revue d'histoire de l'Amérique française, 56,3 (hiver 2003): 303-327.

14. Archives de l'Évêché de Trois-Rivières (à l'avenir AETR), boîte 35, chemise 52-15-9, Rapport sommaire d'une réunion qui a eu lieu au Château de Blois, Trois-Rivières, le 3 février 1953, sous les auspices de la Chambre de commerce, dact., $4 \mathrm{p}$.

15. Jean-Marc Beaudoin, in Le Nouvelliste, 7 juin 1982. Ce journaliste a préparé une série d'articles sur la carrière politique de Joseph-Alfred Mongrain, parus du 24 mai au 7 septembre 1982. 
souscription publique à leur profit est mise sous la responsabilité du comité dit de la Campagne de la Fédération des ouvres, limitée pour l'instant à la seule ville de Trois-Rivières. Composés de personnes nommées par l'évêque, dont certaines sur recommandation des membres de l'autre comité, Conseil et Fédération sont ainsi non seulement intimement liés, mais aussi placés sous l'autorité directe de $\mathrm{M}^{\mathrm{gr}}$ Georges-Léon Pelletier ${ }^{16}$.

\section{2 - UNE ACTIVITÉ SIGNIFICATIVE, MAIS DÉJÀ CONTRARIÉE (1954- 196I)}

Par cette double fondation, préparée de longue main, l’Église trifluvienne se donne les moyens d'une intervention efficace. Soutenus par l'évêque, disposant d'une expertise professionnelle reconnue, d'un important réseau social bénévole et de ressources financières appréciables, Conseil, Fédération puis Caritas-Trois-Rivières seront en mesure d'imprimer de réelles orientations au développement de l'assistance et du bien-être diocésains jusqu'au début des années 1960. Et cela, en dépit de difficultés dont certaines témoignent bien que la société québécoise est déjà en train de changer.

\section{I Une charité "professionnelle»}

Sans surprise, $\mathrm{M}^{\mathrm{gr}}$ Bourgeois devient le directeur administrateur du Conseil central des œuvres. Il le restera jusqu'en 1964. À tous les postes clés, il place ses hommes de confiance. Maurice Laurin, juge à la Cour du bienêtre social, est un ami intime de la famille Bourgeois ; président du conseil d'administration de l'Assistance à l'enfant sans soutien, qui change de nom en 1953 pour celui de Centre de service social du diocèse de TroisRivières, le voilà maintenant président du conseil d'administration du Conseil central des ouvres puis de Caritas-Trois-Rivières, charge qu'il gardera jusqu'à la dissolution de l'organisme en 1966. Depuis 1943, à titre de Grand Chevalier des Chevaliers de Colomb, voire aussi comme président du Club Richelieu en 1952, il a présidé année après année, presque sans exception, la campagne de la Journée de la Livre. Sa renommée et ses réseaux sociaux, il les met désormais au service du nouvel organisme ${ }^{17}$.

16. CM, 100A.0159, Cahier des procès-verbaux...., 7 septembre 1954.

17. Les renseignements biographiques concernant toutes les personnes nommées dans cet article sont consignés dans un fichier nominatif constitué à partir des informations colligées dans plusieurs sources. Les principales sont des centaines d'articles sur les œuvres sociales et caritatives parus dans le journal Le Nouvelliste entre les années 1930 et 1960. Les Rapports annuels de l'Assistance à l'enfant sans soutien puis du Centre de service social et les Listes des décorés pontificaux conservées aux AETR comptent aussi parmi les sources d'information importantes. De plus, mon 
Par ailleurs, Jules Perron en devient le secrétaire exécutif. Membre lui aussi des Chevaliers de Colomb et du Richelieu, ce travailleur social professionnel, fidèle bras droit de Bourgeois, ne craint pas le cumul des tâches: il est secrétaire général de la Journée de la Livre à partir de 1943, secrétaire général de l'Assistance à l'enfant sans soutien à partir de 1946, puis du Centre de service social, secrétaire général de l'Institut psychosocial dès sa fondation en 1947. Deux autres travailleurs sociaux professionnels employés d'abord au Centre de service social sont placés en 1955 par Bourgeois au Conseil central des œuvres, où ils travailleront jusqu'au début des années 1960: Jean-Paul Ramsay, chargé du fonctionnement quotidien ainsi que des relations avec les œuvres et certains bailleurs de fonds, et Gilles Lacroix, qui prend la direction de l'importante commission des œuvres, lieu de rencontre et d'échange entre celles-ci. Quant à Yvon Thériault, à titre de journaliste au Nouvelliste, il est devenu ni plus ni moins au fil des ans le propagandiste des activités de Bourgeois; c'est lui qui fera bientôt du quotidien régional le héraut zélé des campagnes de charité de la Fédération des œuvres, tout en occupant en outre le poste de publiciste au Conseil central puis à Caritas-Trois-Rivières.

Les principaux artisans du Conseil central des œuvres, de la Fédération des œuvres puis de Caritas-Trois-Rivières sont donc des professionnels du service social ou des communications reconnus dans leur milieu et intégrés à plusieurs réseaux sociaux. De plus, $\mathrm{M}^{\mathrm{gr}}$ Bourgeois et Jules Perron animent de près les organismes qui, à l'échelle québécoise et internationale, s'occupent des questions de bien-être. $\mathrm{M}^{\mathrm{gr}}$ Bourgeois est le vice-président de la commission française du Conseil canadien du bien-être social et le président de l'influente commission des "prêtres-directeurs diocésains » de Caritas-Canada, qui réunit les directeurs des centres de services sociaux de tous les diocèses québécois; il est aussi vice-président du Bureau international catholique de l'enfance (BICE), et à ce titre pilote nombre de dossiers au Vatican ${ }^{18}$. Jules Perron préside la commission «enfance» de Caritas-Canada, et Bourgeois l'emmène régulièrement en Europe assister aux congrès annuels du BICE. Pas étonnant dans ces conditions que Caritas-Trois-Rivières multiplie d'abord les réalisations.

collègue Yvan Rousseau m’a donné accès à son fichier sur les «élites» trifluviennes entre 1920 et 1960. Qu'il en soit vivement remercié.

18. ASTR, fonds 0560, boîte 3, CSS-TR, Rapport annuel 1953, 28, et Léon A. Lemay, «Un prêtre d'abord», Le Tourbillon, journal du CSS-CQ, VIII,5 (mars 1981): 1. En 1957, le BICE tiendra son congrès annuel au Québec, en partie à Trois-Rivières; c'est Jules Perron qui en assumera alors le secrétariat général: «Trois-Rivières logera le secrétariat du congrès de 1957», Le Nouvelliste, 9 juin $1956,3$. 


\subsection{Un impact certain sur la planification des services de bien-être et sur le développement des œuvres}

Pour agir sur la conformation et l'orientation des services sociaux et des services d'aide catholiques, le Conseil central, devenu Caritas-TroisRivières en 1959, dispose de deux leviers: sa commission des ouvres et sa capacité de financement.

Deux représentants de chacune des œuvres membres de la Fédération siègent à la commission des œuvres. C'est là qu'ils apprennent à se connaître et à mettre leurs expertises en commun, premières étapes de tout processus de coordination. À partir de 1957, des sous-commissions spécialisées réunissent plusieurs fois par année les œuvres qui interviennent plus spécifiquement dans les secteurs de l'enfance et de la famille, des loisirs, de la santé, pour y favoriser encore davantage le dialogue et entreprendre l'équivalent de ce qu'on appellera plus tard des recherches-actions sur des sujets tels que les ressources sociales disponibles à Trois-Rivières, la délinquance juvénile ou les allocations familiales ${ }^{19}$. Dans tous les cas, il s'agit d'imaginer les moyens d'augmenter le nombre et la qualité des services sociaux offerts à la population de la ville puis du diocèse de Trois-Rivières. Les journées d'étude annuelles représentent une autre occasion de réflexion, de mise à niveau, en vue d'en arriver à une compréhension commune des problèmes sociaux et des manières de les résoudre. Organisées chaque année de 1957 à 1963, elles rassemblent toujours quelques centaines de personnes - 230 en 1957, 200 en 1962 - venues entendre des intervenants de la région et de partout au Québec sur des thèmes comme «le bien-être social dans notre milieu », "l'adolescent vers demain» ou "la planification sociale et son influence sur l'exercice des fonctions de la famille ${ }^{20}$ ».

Enquêtes sur le terrain et colloques annuels conduisent la commission des œuvres à recommander la création d'établissements et de services nouveaux, parfois déjà disponibles à Québec ou à Montréal, plus rarement dans les autres régions. À l’image de ce qui existe dans la métropole, une Société des infirmières visiteuses, par exemple, est fondée en 1955, pour le soin des malades à domicile ${ }^{21}$. Autre exemple, en 1958: à la demande

19. CM, 100A.0159, Cahier des procès-verbaux..., 24 octobre 1955 ; 30 janvier 1958. AETR, boîte 535, chemise 52-15-9, Conseil central des œuvres, Deuxième Rapport annuel, 1955-1956, imprimé, 26 octobre 1956; chemise 52-15-10, Règlement $n^{\circ}$ 3, 30 mai 1960.

20. CM, 100A.0159, Cahier des procès-verbaux..., 30 janvier 1958. Gérald Godin, «Il nous faut prendre conscience de nos responsabilités vis-à-vis [sic] l'adolescent », Le Nouvelliste, 23 mai 1961. Rapport de la cinquième journée d'étude diocésaine de la commission des œuvres de Caritas-Trois-Rivières du diocèse de Trois-Rivières, 13 mai 1961 (Trois-Rivières, 1961), 174 p.

21. CM, 100A.0159, Cahier des procès-verbaux..., 7 juillet, 12 et 24 septembre 1955. 
des cercles Lacordaire du diocèse, le Conseil central accepte de financer un centre de réhabilitation diocésain pour alcooliques, qui travaillera avec le Centre Domrémy de Québec ${ }^{22}$. La même année est ouverte l'École supérieure d'assistance sociale, pour la formation sur place des aides sociaux, un type de formation offert jusque-là seulement à Québec, Montréal et Sherbrooke. Conçue comme un des piliers de l'université que voudrait fonder $\mathrm{M}^{\text {gr }}$ Pelletier, elle sera intégrée au Cégep de Trois-Rivières au moment de sa création, une dizaine d'années plus $\operatorname{tard}^{23}$.

La commission des œuvres n'agit pas seulement pour encourager la fondation d'établissements ou de services nouveaux. Appuyée par la commission des budgets, elle peut aussi provoquer les regroupements d'œuvres existantes. Par exemple, quand la campagne de collecte de fonds est étendue à l'échelle du diocèse, en 1958, le refus de subventionner les conférences paroissiales de la Société Saint-Vincent-de-Paul oblige cellesci, dans les villes qui en sont encore dépourvues, à se doter d'un conseil particulier pour les chapeauter et assurer entre elles une certaine péréquation; Caritas forcera de même les quatre foyers Domrémy du diocèse à se fédérer, en $1963^{24}$.

Le plus souvent toutefois, c'est par le biais des documents qu'elle exige des œuvres pour les accepter comme membres de la Fédération et continuer à les soutenir financièrement que Caritas-Trois-Rivières peut contribuer le plus efficacement à transformer les manières de faire, si elle le juge nécessaire. Beaucoup plus exigeante que les municipalités ou le gouvernement du Québec - qui, dans les années 1950, subventionnent encore bien souvent les organismes de charité, surtout les petits, sans exiger en retour de leur part une quelconque reddition de comptes -, Caritas réclame déjà prévisions budgétaires, programme des activités, rapports d'activités et états financiers, et se réserve le droit d'imposer des modifications aux budgets présentés ${ }^{25}$. Ses moyens lui permettent en outre de financer entièrement certains services privés qu'elle juge essentiels dans un diocèse urbain comme Trois-Rivières et qui ne reçoivent aucune

22. CM, 100A.0159, Cahier des procès-verbaux..., 11 avril 1958.

23. «Étape vers l’enseignement universitaire. École supérieure d’assistance sociale et École de pédagogie ", Le Nouvelliste, 29 mars 1958, 3 et 5. "Une École supérieure d’assistance sociale. Mandement spécial de $M^{\text {gr }}$ Pelletier», Ibid., 4 avril 1958, 3.

24. AETR, boîte 537, chemise 52-15-10, Jean-Paul Ramsay, Étude sur les possibilités d'extension de la Campagne de la Fédération des cuvres de charité, dact., 2 vol., 1958. CM, 100A.0168, Cahier des procès-verbaux des réunions du conseil d'administration de Caritas-Trois-Rivières, 2 mai 1963.

25. CM, 100A.0159 et 100A.0168, Cahier des procès-verbaux..., $1^{\text {er }}$ octobre 1957, 27 août 1958, 3 mars 1960 . 
aide spéciale du gouvernement et encore moins d'un public désapprouvant leurs bénéficiaires: c'est le cas du Service social Sainte-Marie, qui offre aux mères célibataires les services de travailleurs sociaux professionnels, et celui du Foyer Saint-Joseph, qui prend la relève du Gîte dans l'accueil des vieillards indigents et autres sans-abri ${ }^{26}$.

Parmi les premières réalisations de Caritas-Trois-Rivières, il ne faudrait pas oublier non plus le Noël du Pauvre, un Téléthon promis à un avenir durable et dont la première édition est présentée en 1959 sur les ondes de CKTM-TV. D’abord limitée aux villes de Trois-Rivières, Cap-de-la-Madeleine, Shawinigan, Grand-Mère et Saint-Tite, où l'on recueille plusieurs milliers de dollars en quelques heures, l'initiative s'étend sans tarder à Louiseville, puis déborde même des frontières de la Mauricie ${ }^{27}$.

Au total, dans la deuxième moitié des années 1950 et même au tout début des années 1960, l'action du Conseil central puis de Caritas permet réellement de consolider et d'élargir l'éventail des services d'aide et des établissements de services sociaux offerts à la population du diocèse. À côté d'initiatives qui, même si elles utilisent des moyens de collecte nouveaux comme la télévision, restent d'inspiration tout à fait traditionnelle - c'est le cas du Téléthon-, d'autres, telle la création de l'École supérieure d'assistance sociale, annonce déjà des orientations qui seront conservées quand l’État prendra le contrôle de la régulation du système d'assistance et de bien-être social.

\subsection{Les facteurs de succès:}

\section{la mobilisation des élites et le soutien populaire}

L'organisme doit beaucoup ces succès à sa capacité de mobiliser les élites et de stimuler la générosité populaire.

Autour de l'organisme, en effet, gravite un milieu social très bien typé : celui des «élites traditionnelles», sur lesquelles l’Église a l'habitude de s'appuyer pour la charité. Les trente-trois personnes membres entre 1954 et 1961 du conseil d'administration ou des divers comités sont des com-

26. CM, 100A.0159, Cahier des procès-verbaux ..., 29 décembre 1954, 21 décembre 1955. En entrevue avec l'auteure le 17 février 2004, Claude Lemay, qui fut employé de Caritas-Trois-Rivières à partir de 1965 avant de devenir le premier directeur général du Conseil régional de bien-être de la Mauricie en 1968, expliquait que les solliciteurs pour la campagne annuelle de Fédération des œuvres de charité ne mentionnaient jamais que le Service social Sainte-Marie comptait parmi les organismes membres, car plusieurs diocésains refusaient alors de souscrire.

27. Le Noël du Pauvre est une idée de l'abbé Gilles Boulet, qui a été réalisée par Jean-Paul Ramsay de Caritas-Trois-Rivières. Ces propos de Claude Lemay sont confirmés par les sources. CM, 100A.0168, Cahier des procès-verbaux..., 3 février et 23 décembre 1960; et CM, 240.23, Le Noël du Pauvre, historique, s.d., dact., 8 p. 
merçants établis depuis longtemps, des avocats, un juge, des médecins parmi lesquels des spécialistes occupant des fonctions importantes dans les deux hôpitaux de la ville, le gérant de la principale caisse populaire de Trois-Rivières. Leurs réseaux sociaux se ramifient d'autant plus profondément dans la Chambre de commerce, la Société Saint-Jean-Baptiste, le club Richelieu ou les Chevaliers de Colomb que plusieurs d'entre eux y ont assumé ou y assument encore des charges importantes, celle de président le plus souvent. Tous se sont donnés depuis longtemps et continuent de se donner dans les œuvres: à reconstituer leur parcours de bénévoles, on finit par rencontrer tous les établissements et organismes de charité de la ville. Au cours des années 1950, plusieurs d'entre eux sont d'ailleurs élevés au rang de Commandeur de l'Ordre de Saint-Grégoire le Grand, de l'Ordre de Saint-Sylvestre, de l'Ordre équestre du Saint-Sépulcre ou encore reçoivent la croix «Pro Ecclesia et Pontifice», en signe de la reconnaissance de l'Église. C'est Madame Thérèse Denoncourt, par exemple: elle et son mari chirurgien offrent des distractions aux chanoines, qu'ils invitent régulièrement à leur chalet ${ }^{28}$. Ou Aimé Montcalm, concessionnaire de Chevrolet, qui fournit des automobiles aux prêtres de l'évêché. Ou encore Robert Durand, le gérant de la caisse populaire, très lié au chanoine Robert Giroux, procureur du diocèse. Parmi les administrateurs de l'organisme se trouvent aussi les docteurs Jean-Baptiste Leblanc, Jean Gascon et Conrad Godin qui soignent le plus souvent gratuitement les orphelins des Dominicaines du Rosaire, les patients du Dispensaire Notre-Dame de la Paix ou les mères célibataires les plus démunies de l'Hôpital Sainte-Marie. Puis encore le futur maire Laurent Paradis et l'ancien maire Arthur Rousseau, celui-ci entrepreneur de pompes funèbres tout à côté du grand parc qui s'étend devant la cathédrale; le boucher-épicier Ubald Laforest, qui a toujours refusé de demander le permis de vente de bière, échevin du quartier Notre-Dame et adversaire coloré du maire Mongrain lors de la campagne électorale municipale de $1953^{29}$; et enfin, l'avocat François Nobert, échevin du quartier SaintLouis.

Quand la campagne annuelle de charité prend une ampleur diocésaine à partir de 1958, les officiers recrutés hors de Trois-Rivières présentent presque le même profil socioprofessionnel et bénévole, un peu atténué cependant, puisque les autres villes et gros bourgs du diocèse n'offrent

28. Ce détail nous a été révélé en entrevue par Claude Lemay.

29. Réjean Hould et Mario Audet, «Pattes de velours et griffes pointues», Les anecdotes politiques de J.-A. Mongrain (Trois-Rivières, [s.n.], 1990), 41. 
pas un éventail aussi prestigieux de lieux d'insertion sociale. Parmi eux, on trouve, entre autres, un médecin, un pharmacien, deux comptables agréés, le gérant de la Banque canadienne nationale de Shawinigan, deux courtiers d'assurances ainsi que deux chimistes industriels.

Arrive l'automne, l'époque de la campagne de souscription, le moment le plus intense de l'année. Au bureau, on a systématisé durant les mois précédant la confection du fichier des donateurs, conçu à l'époque de la Journée de la Livre; on a stimulé saison après saison l'intérêt pour les œuvres en expédiant à deux mille personnes le feuillet trimestriel Vie des œuvres et en diffusant dans les journaux, à la radio et à la télévision des bulletins réguliers d'informations se rapportant au bien-être social; on a procédé au moins une fois à un sondage pour évaluer la manière de mener la campagne et solliciter des suggestions pour l'améliorer ${ }^{30}$. Profitant entre autres de l'expertise du Conseil canadien du bien-être social et de CaritasCanada, on a mis sur pied un système pyramidal de collecte très sophistiqué, basé surtout sur la sollicitation paroissiale, mais qui comprend aussi des quêtes spéciales auprès des industriels et commerçants, du clergé, et des corporations municipales et scolaires ${ }^{31}$. On a recruté un président connu, crédible, influent: Laurent Paradis en 1954, Arthur Rousseau en 1955, le dentiste et homme public François-Xavier Lacoursière en 1956, Aimé Montcalm en 1957, puis, quand la campagne prend une envergure diocésaine, des personnalités provenant tour à tour des autres villes de la Mauricie. Enfin, la campagne est lancée en grande pompe: message de l'évêque, nombreuses conférences radiophoniques du président de l'année patronnées par le poste CFTR, multiplication des interventions publiques. En 1954, Le Nouvelliste publie 87 articles entre le 14 et le 30 novembre, 117 messages radios sont diffusés, 12000 circulaires distribuées et 35 conférences prononcées par les membres du conseil d'administration dans leurs différents milieux; en 1956, on relève 140 articles de journaux entre le 28 octobre et le 17 novembre, 220 appels à la radio, 17000 feuillets, 400 affiches et pancartes, 4000 autocollants pour autos et fenêtres, 20000 papillons de Caritas $^{32}$. Les solliciteurs se comptent par centaines : 425 en

30. CM, 100A.0159, Cahier des procès-verbaux..., 11 septembre 1957, 30 janvier 1958. La collection de Vie des cuvres est conservée aux AETR.

31. CM, 100A.0159, Cahier des procès-verbaux..., 27 octobre 1954. AETR, boîte 535, chemise 52-15-7, Plan d'organisation paroissiale, Trois-Rivières, Caritas-Trois-Rivières, 1959 [?], imprimé, 20 p.; Plan d'organisation, Section Clergé, Campagne de la Fédération des œuvres de charité du diocèse de TroisRivières, dact., $6 \mathrm{p}$.

32. ASTR, fonds 0560 , boîte 2 . Cette boîte contient les spicilèges de $\mathrm{M}^{\mathrm{gr}}$ Bourgeois sur les campagnes annuelles de charité, gros cahiers constitués chacun de tous les articles de presse 
1954, puis 600, puis 723 ; en 1958, la campagne s'étend aux principales villes, en 1959, à tout le diocèse : le nombre de solliciteurs bondit à 3000, à 5000 ! Un tel effort produit des résultats : de 87637 \$ en 1954 à $173713 \$$ en 1960. Autant d'argent à distribuer à quinze œuvres d'abord, puis jusqu'à vingt-six, en $1960^{33}$.

Malgré les réels succès des campagnes de charité et les moyens qu'ils donnent à Caritas pour orienter et planifier les services sociaux et les services d'aide à l'échelle du diocèse, la mobilisation des élites et le soutien populaire ne réussissent toutefois pas à masquer le fait que la charité catholique connaît, déjà, quelques difficultés.

\subsection{Les premières difficultés : l'hégémonie du \\ Centre de service social, la résistance des œuvres, un certain retard sur un milieu en pleine transformation}

Première difficulté, l'organisme a du mal à se démarquer du Centre de service social, qui lui a pratiquement donné naissance. S’agit-il de créer des établissements nouveaux? La Société des infirmières visiteuses fonctionne d'abord en vertu de la charte civile du CSS $^{34}$. L’École supérieure d'assistance sociale, pour sa part, prend au fond la relève des cours de perfectionnement qu'offrait l'Assistance à l'enfant sans soutien depuis 1951 ; à sa création en 1958, son directeur général, Gilles Lacroix, les directeurs de ses trois départements, sept des membres de son conseil d'administration dont $\mathrm{M}^{\mathrm{gr}}$ Bourgeois, et neuf de ses quinze professeurs font partie du Centre de service social ${ }^{35}$ ! Bourgeois, qui souhaite "éliminer les mendiants professionnels et les exploiteurs de la charité», impose en outre à Caritas une fonction de contrôle social des personnes assistées qui conduit son personnel à investir une énergie considérable, pendant des années, pour établir et maintenir un fichier central des œuvres et un fichier de Noël que celles-ci n'entretiennent que très mal, faute de secrétariat et d'intérêt, et auquel la Société Saint-Vincent-de-Paul refusera même toujours, par principe, de communiquer quelque information que ce soit ${ }^{36}$. Enfin, tout

publiés par les différents journaux. Les références y sont extrêmement nombreuses, provenant surtout du Nouvelliste, mais aussi du Bien public et même de L'Action catholique, un journal de Québec.

33. CM, 100A.0159, Cahier des procès-verbaux..., 21 octobre 1955, 4 septembre 1956, 27 août 1957, 19 et 28 janvier 1959; CM, 100A.0168, Cahier des procès-verbaux..., 21 décembre 1959, 11 décembre 1960 .

34. ASTR, fonds 0560, boîte 3, CSS-TR, Rapport des activités pour l'année 1955, 24.

35. ASTR, fonds 0560 , boîte 3, CSS-TR, $26^{\mathrm{e}}$ Rapport annuel, 1959 «Pour la rédemption sociale».

36. CM, 240.21, Fichier central des œuvres. Rapport des visites de Mlle Beaudoin aux personnes cidessus mentionnées, 23 mars au 17 avril 1959, dact., non paginé, 12 p. Le fichier central des œuvres 
en ayant sans cesse réclamé et obtenu, depuis les années 1930, une augmentation du financement public pour les établissements de bien-être appartenant à l'Église, $\mathrm{M}^{\mathrm{gr}}$ Bourgeois est viscéralement opposé à tout contrôle étatique de leurs activités et de leur gestion: pour assurer l'indépendance de son Centre de service social, il réclame donc une large part du produit de la campagne annuelle de charité ${ }^{37}$. En 1958, par exemple, il «fait remarquer que la première Campagne diocésaine a connu du succès surtout à cause des activités du Centre de service social en dehors de TroisRivières. Il émet le vœu que ce fait sera considéré lorsqu’il présentera une demande d'octroi au sous-comité des budgets ${ }^{38}$ ». Au total, le Centre de service social retire la part du lion, au point qu'il faut décider en 1960 de la restreindre à $20 \%$ du produit de la campagne ${ }^{39}$.

L'existence de Caritas-Trois-Rivières remet aussi en cause bien des pratiques. Jusqu'en 1954, petites œuvres comme gros établissements d'hébergement ont été habitués à mener leur barque à leur guise. Voilà que, en contrepartie du soutien financier qu'ils obtiennent sans avoir à le rechercher par leurs propres moyens, ils doivent renoncer au moins partiellement à leur autonomie, s'empêcher de mener toute autre sollicitation publique, accepter une ingérence directe dans leur gestion interne, leurs projets, leur budget et se soumettre à des exigences typiquement bureaucratiques comme de remplir des formulaires et se rendre à des convocations! Certains se montrent peu dociles. L'Association des infirmes, véritable épine au pied de Caritas pendant plusieurs années, continue par exemple de s'adresser aux conseils des villes de Trois-Rivières et de Cap-de-la-Madeleine pour en obtenir une aide particulière ${ }^{40}$. La Société Saint-Vincent-de-Paul, pour sa part, sait que c'est en partie sur son nom que se fait la campagne annuelle de souscription: elle exige $20 \%$ de la recette. Puis ce sont les membres du conseil d'administration euxmêmes qui plaident pour l'œuvre qu'ils patronnent plus particulièrement:

est en fait un fichier nominatif des personnes assistées. C’est seulement en 1966 que Caritas, dépitée, renoncera au fichier central, «dû au fait que les agences de bien-être du diocèse ne voient pas l'importance d'un tel service», Fichier central des œuvres, document sans date, non paginé, après le 2 mai 1966.

37. Ibid., 16 février 1955, 11 septembre 1957, 23 décembre 1960. La part de la SSVP descendra à $15 \%$ du produit à partir de 1961 .

38. Ibid., 24 novembre 1958.

39. CM, 100B.04C73, Cahier des procès-verbaux du comité des budgets, du 18 mars 1959 au 28 mars 1973, 6 décembre 1960, 21 novembre 1963. La part du CSS remontera à $33 \%$ du produit en 1963.

40. CM, 100A.0159 et 100A.0168, Cahiers des procès-verbaux..., 6 avril 1955, 19 avril 1956, 24 mai 1961, entre autres dates. 
quand le juge Laurin ne tarit pas d'éloge sur le Foyer Saint-Joseph, difficile de ne pas augmenter le montant qu'on lui avait d'abord consenti ${ }^{41}$ ! Bref, l'argent ne permet pas tout, et l'individualisme traditionnel des ouvres constitue une autre limite à la marge de manœuvre de Caritas dans ses fonctions innovatrices de planification et de coordination. Cette marge se restreint encore à partir de 1958, puisque les principaux organisateurs de la campagne dans les autres villes du diocèse réclament que l'argent ramassé chez eux soit versé à des œuvres locales ${ }^{42}$.

Soumise à l'hégémonie du Centre de service social et aux prises avec les œuvres, qui acceptent mal de transformer leurs pratiques traditionnelles, Caritas doit aussi compter avec tout le milieu ambiant. Or ce milieu, culturellement, est en train de se transformer plus vite qu'elle.

Jusqu'à un certain point, les membres du conseil d'administration s'en rendent compte et essaient de corriger le tir. Ces notables voient bien, par exemple, qu'ils ne reflètent pas les forces vives de la ville puis du diocèse, touchés dans les années 1950 par un véritable boom industriel. Pourraiton s'adjoindre un dirigeant d'un des syndicats catholiques? «Après discussion, il est résolu à l'unanimité de laisser cette question en suspens, vu qu'elle comporte de grandes difficultés ${ }^{43}$.» On n'y reviendra jamais. Trouvera-t-on le représentant des «catholiques irlandais » que le règlement du Conseil central prévoit qu'il faut nommer? Le premier industriel pressenti refuse, puis l'arrimeur Desmond Malone accepte; mais peut-on encore qualifier d'irlandais celui qui ne l'est que par un grand-père établi à TroisRivières au XIX ${ }^{\mathrm{e}}$ siècle ${ }^{44}$ ! Il faudrait bien, enfin, augmenter le nombre des hommes d'affaires au sous-comité des budgets, afin d'en rehausser «la compétence, la valeur et le prestige ${ }^{45}$ ", un autre souhait qui restera lettre morte. Les administrateurs de Caritas jugent aussi qu'un respect trop strict et, même à leurs yeux, dépassé de la confessionnalité limite leur capacité

41. CM, 100A.0159, Cahier des procès-verbaux..., 2 février 1956. Ce n'est qu'un exemple d'une situation qui s'est répétée pour d'autres œuvres.

42. Déjà, en 1954, un groupe d'hommes d'affaires de Cap-de-la-Madeleine avait tenté d'établir une Fédération des œuvres locales, «pour éviter que des sommes d’argent versées par des citoyens du Cap servent aux œuvres de Trois-Rivières »; mais les curés étant restés solidaires de $\mathrm{M}^{\mathrm{gr}}$ Bourgeois, le projet avorta. AETR, boîte 535, chemise 52-15-2, Lettre de $M^{\mathrm{gr}}$ Bourgeois à $M^{\mathrm{gr}}$ Pelletier, 15 octobre 1954. Le procès-verbal d'une réunion à laquelle le curé de Saint-Lazare avait convoqué ses confrères des autres paroisses de Cap-de-la-Madeleine est joint à la lettre.

43. CM, 100A. 0159, Cahier des procès-verbaux..., 14 octobre 1954.

44. Ibid., 23 septembre et 14 octobre 1954, et 16 mars 1955. Pour la biographie de Desmond Malone, voir: Société historique industrielle inc., Une page d'histoire de Trois-Rivières et la région (Trois-Rivières, s.é., 1955), 148.

45. Ibid., $1^{\mathrm{er}}$ octobre 1957. 
d'information du public, de coordination des œuvres et de pénétration dans le monde de l'industrie. Mais si $\mathrm{M}^{\text {gr }}$ Pelletier daigne agréer que le Conseil central puisse s'affilier au Conseil canadien de bien-être social, un organisme neutre, en revanche il refuse carrément que le Bottin des æuvres $\mathrm{du}$ diocèse contienne les noms des associations philanthropiques non catholiques, et même que le président de la campagne, tout bon catholique soit-il, appartienne à un club neutre, tels le Rotary, le Kiwanis ou les Optimistes ${ }^{46}$. Pourtant de tels clubs recrutent beaucoup de membres parmi les Canadiens français, de moins en moins enclins à limiter leur vie associative aux mouvements mis sur pied par l'Église.

En cette deuxième moitié des années 1950, un autre phénomène commence à se faire sentir: les œuvres catholiques doivent affronter la concurrence nouvelle d'un grand nombre d'organismes à vocation pancanadienne, très visibles et si riches qu'ils peuvent payer leurs solliciteurs à commission, plutôt que de devoir s'appuyer sur des bénévoles: en février 1956, l'Institut national canadien pour les aveugles rafle 22000 \$ à l'échelle du diocèse, la Croix-Rouge $30000 \$$ le mois suivant, tandis que la Société du cancer recueille $8000 \$ \$^{47}$, autant d'argent qui aboutit le plus souvent en Ontario, dans l'indifférence générale. La campagne de la Croix-Rouge et celle pour les aveugles sont dirigées par des militaires, qui peuvent puiser dans un budget spécial de l'armée, pour la publicité notamment. Des personnalités majeures de la région s'en font les porte-parole, qui ont bien souvent en commun d'appartenir au Parti libéral: Édouardina Dupont, par exemple, est active dans les sections trifluviennes de la Société canadienne du cancer et de l'Institut national canadien pour les aveugles ${ }^{48}$. Si l'on ajoute que les grandes entreprises, gérées par des anglophones, se désintéressent presque complètement de la charité catholique du diocèse, alors que leurs sièges sociaux, à Montréal, donnent généreusement aux organismes philanthropiques et aux ouvres protestantes et juives, on conçoit que les objectifs des campagnes demeurent modestes, afin que la Fédération puisse les atteindre: celui de la campagne de 1957, par exemple, est fixé à 94315 \$ plutôt qu’à 160957 \$, ce qui serait pourtant à la fois plus conforme aux besoins et plus en rapport avec la moyenne

46. Ibid., 14 octobre et 30 décembre 1954, 16 février 1955. AETR, Jean-Paul Ramsay, op. cit., 31.

47. AETR, Jean-Paul Ramsay, op. cit., appendice 3, 77-78. Entrevue avec Claude Lemay, 17 février 2004, pour l'information sur la commission payée aux solliciteurs.

48. Société historique industrielle inc., Une page d'histoire de Trois-Rivières et la région (TroisRivières, s.é., 1955). Édouardina Dupont est l'une des têtes dirigeantes de l'Association des femmes libérales. 
des soixante-dix-sept villes canadiennes qui organisent des campagnes groupées, «because a certain number of individuals and companies have not subscribed sufficiently in the past years", comme l'écrit le président de la campagne aux dirigeants des grandes entreprises ${ }^{49}$. Les centaines, voire les milliers de solliciteurs ne peuvent donc faire oublier aux organisateurs des campagnes de la Fédération qu'ils travaillent dans un milieu où les clivages sociaux, politiques et ethniques se conjuguent et se renforcent, limitant ainsi les succès de la charité catholique diocésaine.

Enfin, dernier signe avant-coureur des prochains bouleversements, les pouvoirs publics se font de plus en plus tirer l'oreille. Certes, la Ville de Trois-Rivières donne $15000 \$$ en 1954 et jusqu'à 18500 \$, en 1957, à la campagne de la Fédération, assurant ainsi chaque fois le dépassement de l'objectif fixé. Cette année-là, toutefois, certains échevins s'opposent avec succès à la requête de la délégation de Caritas, venue demander à la Ville une contribution plutôt de l'ordre de 20000 \$; en cette période de fort chômage, ils préfèrent augmenter le budget attribué au Service municipal de bien-être. Ce retour à la politique du maire Mongrain précède même son propre retour à la mairie, en 1960, qui signe le désengagement progressif de la Ville à l'égard de la charité catholique ${ }^{50}$. À partir de 1958, les résultats des campagnes annuelles de la Fédération resteront toujours en deçà de l'objectif fixés1.

49. CM, 100A.0159, Cahier des procès-verbaux..., 13 septembre 1956. AETR, boîte 535, chemise 52-15-2, Copie d'une lettre de Aimé Montcalm, président de la campagne de la Fédération des auvres, à C. D. Jentz, manager, St. Lawrence Corp. Ltd, 10 octobre 1957. Ce phénomène d'indifférence du patronat anglophone pour les œuvres de bien-être des petites villes francophones du Québec avait déjà été remarqué par Everett C. Hughes, Rencontre de deux mondes. La crise d'industrialisation du Canada français (Montréal, Boréal Express, 1972), 225, première édition 1945. Il demeure constant: en 1965, encore, les organisateurs de la campagne trifluvienne constateront que pour 1964, «la contribution financière du monde des affaires s'est établi [sic] à $10 \%$ pour le secteur Commerce et à $10 \%$ pour celui Industrie. Par contre, à Montréal, $60 \%$ du montant de la Campagne est venu des entreprises commerciales et industrielles». Voir: AETR, boîte 535, chemise 52-15-7, Nos chefs d'entreprises et la Fédération des œuvres, dact., 2 p. ; il s'agit d'un argumentaire dressé à l'intention des solliciteurs.

50. Yvon Thériault, Trois-Rivières incorporée, 1857-1957 (Trois-Rivières, s.é., 1958), 87 p. La crise économique de 1957 continue de se faire sentir dans les années suivantes, ce qui pèse lourd sur le budget du Service de bien-être municipal, dont l'une des fonctions est de secourir les sansemploi. Voir Le Nouvelliste, 6 novembre 1959. Pour des exemples des difficultés de Caritas à se faire verser les sommes consenties par la Ville de Trois-Rivières, voir entre autres: CM, 100A.0159, Cahier des procès-verbaux..., 19 novembre et 17 décembre 1956, 12 décembre 1957, 28 janvier 1958, et CM 100A.0168, Ibid., 23 novembre 1959. En 1965, le conseil municipal de Cap-de-la-Madeleine informera à son tour Caritas de sa décision de lui retirer graduellement son soutien financier: Ibid., 16 mars 1965 .

51. En 1958, la campagne rapporte 98,2\% de l'objectif, fixé à $175000 \$$. En 1959, on recueille 187000 \$ des 203000 \$ espérés et en 1960, 173713 \$ sur un objectif de 205000 \$. CM, 100A.0159 et 100A.0168, 19 janvier et 23 novembre 1959, 12 décembre 1960. 
La première partie de l'existence de Caritas-Trois-Rivières se clôt donc sur un bilan mitigé : des réalisations certes, une mobilisation des élites et de la capacité de charité de la population, mais des limites réelles à son influence sur la coordination des œuvres et la planification des services sociaux diocésains.

\section{3 - UN DÉCLIN INEXORABLE (196 I - 1 966)}

À partir de 1961, le contexte change radicalement. Pour le dire en trois mots, $\mathrm{M}^{\mathrm{gr}}$ Pelletier remet en cause la participation de Caritas-TroisRivières à l'assistance et au bien-être; parallèlement, l'État québécois de la Révolution tranquille affirme de plus en plus nettement sa présence dans le vaste domaine des affaires sociales; tandis que toutes sortes de nouveaux organismes à vocation de conseil de recherche ou de lobby sont fondés, principalement par les travailleurs sociaux professionnels, pour tenter d'orienter les décisions du gouvernement Lesage. Malgré quelques nouvelles réalisations, la conjonction de ces trois facteurs vide à brève échéance Caritas-Trois-Rivières de ses forces vives et de sa pertinence. L’organisme disparaît en 1966.

\section{I La perte du soutien épiscopal}

$\mathrm{M}^{\mathrm{gr}}$ Pelletier finit-il par prendre ombrage de l'influence qu'exerce $\mathrm{M}^{\mathrm{gr}}$ Bourgeois sur l'orientation du bien-être dans le diocèse de Trois-Rivières? Désiret-il limiter tant soit peu la liberté d'action dont jouit son encombrant confrère, entre autres du fait que celui-ci réussit à faire aboutir vers ses œuvres de prédilection une partie importante des fonds versés par Caritas à même les revenus des campagnes annuelles de souscription? En 1960, par exemple, une fois défalqué ce que garde l'organisme pour son propre fonctionnement, c'est presque $45 \%$ des sommes octroyées qui sont versées au Centre de service social, à l'Institut psychosocial et aux trois orphelinats des Dominicaines du Rosaire ${ }^{52}$. Toujours est-il que l'évêque décide en 1961 d'organiser une campagne diocésaine et paroissiale triennale en faveur de ses propres cinq grands projets : la construction d'un Grand Séminaire et d'un nouvel évêché, celle d'une maison centrale de l'Action catholique diocésaine, l'agrandissement du Séminaire Sainte-Marie à Shawinigan et la

52. CM, 100A.0168, Cahier des procès-verbaux..., 23 décembre 1960. Caritas-Trois-Rivières verse cette année-là 169865 \$. Sur ce montant, elle garde $25660 \$$ pour ses frais de fonctionnement et ceux de la campagne de la Fédération. Des 135705 \$ restants, $34000 \$$ vont au CSS, $5000 \$$ à l'Institut psychosocial, $10000 \$$ à Ville-Joie Saint-Dominique, $7000 \$$ au Patronage Saint-Charles et 5000 \$ à Ville-Joie du Rosaire, pour un total de $61000 \$$. 
réfection de la cathédrale ${ }^{53}$ : rien de social, donc. Et la sollicitation se fera essentiellement dans les paroisses, ce qui revient à scier la branche sur laquelle est assise la campagne de la Fédération, puisque celle-ci recueille précisément dans les paroisses la plus grande partie des dons des Mauriciens. Une entente avec l'évêque limite les dégâts en 1961, mais ensuite les revenus de la campagne de charité se ressentent de la double sollicitation diocésaine: ils se maintiennent autour de $143000 \$$ en 1962 et 1963, puis remontent à environ $152000 \$$ en 1964 et 1965, après la fin de la campagne pour les projets de $\mathrm{M}^{\mathrm{gr}}$ Pelletier ${ }^{54}$. C'est donc beaucoup moins qu’à la fin des années 1950, alors que les besoins sociaux, eux, ne diminuent pas. Pour tenter de contrer le mouvement, les administrateurs ne tarissent pas de suggestions. En vue de la collecte de fonds de 1963, Robert Durand, le persévérant ${ }^{55}$ président de la commission des budgets, réussit à obtenir de $\mathrm{M}^{\mathrm{gr}}$ Pelletier qu'il consente à ce que quelques hommes d'affaires protestants approchent tout spécialement les industriels pour les inciter à donner! On se rend même à l'idée d'inclure des femmes et des jeunes dans les comités de campagne de 1964! À partir de 1964, d'ailleurs, on met sur pied des comités paroissiaux permanents, dans les villes et villages de tout le diocèse, dans l'espoir d'obtenir un meilleur succès ${ }^{56}$.

\subsection{Dans l'orbite de l'État}

Tout cela affecte bien sûr la capacité d'agir de Caritas-Trois-Rivières. C'est donc presque avec soulagement que l'organisme constate la participation croissante de l'État au financement des œuvres: «Il existe dans notre milieu une tendance poussée vers l'étatisation du bien-être social. Nos œuvres membres reçoivent une part croissante de leur budget des gouvernements ${ }^{57}$.» Désormais, Caritas entreprend même parfois des démarches spéciales auprès de tel ou tel service ministériel pour obtenir un

53. Campagne diocésaine et paroissiale de Trois-Rivières, 1961 (Trois-Rivières, Évêché, 1961), imprimé, 28 p. «Pour réaliser cinq projets diocésains importants. Grande campagne de souscription diocésaine et paroissiale inaugurée officiellement», Le Nouvelliste, 21 juin 1961. Ces projets ont tous été réalisés, sauf la construction d'un nouvel évêché; à la place, on a toiletté de fond en comble celui bâti sous $\mathrm{M}^{\mathrm{gr}}$ Laflèche.

54. CM, 100A.0168, Cahier des procès-verbaux..., 20 décembre 1961, 20 mars et 27 décembre 1962, 26 janvier 1964, 31 janvier 1965. AETR, boîte 535, chemise 52-15-9, Louis-Georges Pérusse, président, Rapport de la campagne de 1966, dact. 19 p. (on y donne le résultat pour 1965, dernière campagne menée sous l'égide de Caritas).

55. Robert Durand occupe ce poste de 1954 à 1966, soit tout au long de l'existence de l'organisme.

56. CM, 100A.0168, Cahier des procès-verbaux..., 5 septembre, 21 octobre et 27 novembre 1963. AETR, boîte 535, chemise 52-15-10, Règlement n ${ }^{\circ}$ 4, février 1964.

57. CM, 100A.0168, Cahier des procès-verbaux...., 27 décembre 1962. 
soutien, au moins temporaire, pour l'une ou l'autre d'entre elles ${ }^{58}$. Quel changement par rapport à la philosophie qui l'animait dans les années 1950 ! Il faut dire que grâce à l'augmentation du financement public, l'organisme peut diminuer en 1962 les montants consentis à celles qui bénéficient du soutien de l'État sans mettre les œuvres en danger. Elle peut ainsi continuer à offrir le même soutien financier à celles qui dépendent entièrement de la campagne de la Fédération, comme le Foyer Joly destiné aux mères célibataires ${ }^{59}$. Seul $\mathrm{M}^{\mathrm{gr}}$ Bourgeois refuse cette logique de remplacement. Comme il a bon espoir que le ministère verse au Centre de service social une somme égale à celle remise par Caritas, il demande au comité des budgets de réserver désormais au CSS non plus $20 \%$, mais bien $33 \%$ de la recette annuelle : après tout, s'exclame-t-il, «Il faut quand même sauvegarder l'initiative privée ${ }^{60}$ !»

En fait, c'est en grande partie parce que le Dispensaire Notre-Dame de la Paix disparaît en 1962, après l'instauration du régime d'assurance-hospitalisation qui rend ses services obsolètes ${ }^{61}$, parce que Ville-Joie Providence et Ville-Joie du Rosaire ferment leurs portes en 1963 et 1966, quand l'État encourage désormais systématiquement le placement des jeunes enfants dans des foyers nourriciers plutôt qu'en institution, parce que Ville-Joie Saint-Dominique ou le Patronage Saint-Charles hébergent pour la même raison de moins en moins d'enfants et d'adolescents, et enfin parce qu'elle puise largement dans la réserve que lui a créée l'entente de 1961 avec l'évêché, que Caritas réussit à maintenir sa contribution au budget de nombreux petits services d'aide, tels les Villages étudiants, le Camp des enfants infirmes, les foyers Domrémy, les sociétés des infirmières visiteuses de Trois-Rivières et de LaTuque, les Dames charitables de Grand-Mère et de Louiseville, le Foyer Saint-Joseph ou encore le Foyer Joly. On le voit, l'organisme devient un acteur de plus en plus marginal sur la scène du bien-être diocésain. Au point même qu'en 1964, la Société Saint-Vincentde-Paul, qui n'a accepté l'autorité de Caritas qu'à contrecœur et seulement

58. CM, 100B.04C73, Procès-verbaux du comité des budgets..., 28 novembre 1961. Une telle démarche auprès du Service des personnes âgées du ministère de la Famille et du Bien-être social est entreprise en 1961 au bénéfice du Foyer Saint-Joseph.

59. CM, 100A.0168, Cahier des procès-verbaux..., 12 et 27 décembre 1962.

60. CM, 100B.04C73, Cahier des procès-verbaux du comité des budgets..., 14 novembre 1963. Caritas agrée à cette demande à la réunion suivante de son conseil d'administration, tenue le 21 novembre.

61. ASTR, fonds 0145, Dispensaire Notre-Dame de la Paix, chemise «Procès-verbaux, Procèsverbal de la réunion du Conseil d'administration du Dispensaire Notre-Dame, tenue au presbytère Notre-Dame de la Paix, le 19 novembre 1962. 
parce qu'elle en retirait un bénéfice, finit par se demander si elle continuera de faire partie de la Fédération des ouvres ${ }^{62}$ !

En matière de coordination et de planification aussi, Caritas-TroisRivières est forcée, dans les années 1960, de restreindre beaucoup ses activités. Sa plus grande réalisation de la période demeure sans conteste la mise sur pied, en 1963, du Bureau d'aide légale aux nécessiteux, sorte de préfiguration de ce qui deviendra en 1972, sous l'égide de l'État, le système d'Aide juridique. Grâce à ce service entièrement financé par l'organisme, les diocésains à faible revenu peuvent désormais recevoir des avis juridiques et bénéficier des services d'un avocat pour ester en justice dans toutes sortes de situations aussi variées que la séparation des époux et la garde des enfants, les relations entre parents et enfants en cas d'insubordination ou de mauvais traitements, les pensions de vieillesse, les faillites personnelles ou les rapports entre propriétaires et locataires ${ }^{63}$. Cependant, pour une telle réussite, combien de projets ne verront pas le jour! Il faudrait, par exemple, convaincre les commissions scolaires d'offrir les services de travailleurs sociaux dans les écoles; mais rien, finalement, ne semble avoir été fait en ce sens ${ }^{64}$. Non, seule la coordination des vestiaires et des soupes populaires semble maintenant à portée réelle de Caritas-Trois-Rivières ${ }^{65}$.

En fait, à partir de 1961, l'organisme se contente le plus souvent de mener des études, qu'elle remet d'ailleurs non plus seulement à l'évêché, mais aussi au ministère de la Famille et du Bien-être social. C'est ce qui se produit en 1962, avec le rapport du comité chargé d'étudier le projet de transformation de l'ancien Collège Séraphique des franciscains, à Trois-Rivières, en un foyer pour couples âgés. Parfois même, Caritas invite des représentants du ministère à devenir membre tout de suite du comité d'étude: c'est le cas en 1963, pour la préparation de l'enquête sur le Patronage Saint-Charles ${ }^{66}$.

62. Pour tout ce qui concerne ce paragraphe, voir CM, 100A.0168, Cahier des procès-verbaux..., 12 décembre 1961, 27 décembre 1962, 27 novembre 1963, 16 décembre 1964, 21 février 1966; aussi CM, 100B.04C73, Cahier des procès-verbaux du comité des budgets, 5 mars 1964. Ces procès-verbaux témoignent souvent, entre 1955 et 1969, des difficultés considérables qu'ont connues avec la Société Saint-Vincent-de-Paul, le Conseil central des œuvres puis Caritas puis les organismes qui ont pris la relève.

63. APETR, fonds Charles-Édouard-Bourgeois, boîte Service social, chemise Assistance publique, Étude des besoins d'un bureau d'aide légale aux nécessiteux, Mémoire présenté au Conseil du Barreau du district de Trois-Rivières par Caritas-Trois-Rivières, novembre 1961, dact., 12 p., annexes. Voir aussi CM 100A. 0168, Cahier des procès-verbaux..., 20 mars et 24 septembre 1962, et 30 janvier 1963.

64. CM, 100A.0168, Cahier des procès-verbaux..., 6 août 1962.

65. Ibid., 100A.0168, Cahier des procès-verbaux..., 20 mars 1962.

66. Ibid., 20 mars 1962, 6 novembre 1963. 
Même si l'organisme ne doit pas un sou de son budget aux fonds publics, ses activités, dans les années 1960, gravitent donc de plus en plus dans l'orbite des initiatives prises par l’État québécois qui, en reprenant à son compte bien des projets imaginés un peu partout au Québec par les conseils d'œuvres diocésains, est en train de s'imposer comme le véritable régulateur du système d'assistance et de bien-être social.

\subsection{Le départ des travailleurs sociaux}

C'est sans doute pourquoi Caritas-Trois-Rivières s'investit encore plus intensément qu'auparavant dans les mouvements extérieurs au diocèse qui s'occupent de bien-être social et tentent tous, désormais, d'influencer les orientations de l'État. En 1962, on retrouve Bourgeois, Ramsay, Lacroix et Perron sinon toujours dans le comité exécutif du Conseil canadien du bien-être social, de Caritas-Canada ou de la nouvelle Corporation des travailleurs sociaux, du moins aux réunions que convoquent de plus en plus régulièrement tous ces organismes ${ }^{67}$. De même, c'est en délégation importante que Caritas-Trois-Rivières et le Centre de service social répondent à l'invitation lancée dès 1960 par le ministre Émilien Lafrance, qui réunit chaque année à Québec les représentants de son ministère de la Famille et du Bien-être social, ceux des conseils d'œuvres et des agences diocésaines de service social, ainsi que ceux des services de bien-être des municipalités pour établir un dialogue permanent entre les acteurs du bien-être social au Québec ${ }^{68}$. Quand les agences sociales diocésaines se regroupent, en 1963, en une Fédération des services sociaux à la famille pour tenter de convaincre le gouvernement du Québec de ne pas s'immiscer dans le champ du service social familial, où elles souhaitent continuer d'intervenir selon leurs propres priorités ${ }^{69}$, c'est Gilles Lacroix qui occupe le premier la présidence de l'organisme. Par ailleurs, Jules Perron et Gilles Lacroix comptent respectivement parmi les membres fondateurs et premiers administrateurs du Conseil de bien-être du Québec, fondé lui aussi en $1963^{70}$. Caritas-Trois-Rivières et le Centre de service social ont

67. Ibid., 19 mai 1962 .

68. APETR, fonds Charles-Édouard-Bourgeois, boîte Service social, chemise «Les services d'assistance sociale/ 2 (rapports)", Procès-verbal de la réunion des services d'assistance sociale du Ministère de la Famille et du bien-être social, avec les Conseils d'ouvre, les agences, les services municipaux de bien-être, tenue le 4 avril 1962 à l'amphithéâtre de la Faculté de commerce de l'Université Laval, Cité universitaire, Québec, format légal, dact., 38 p.

69. Gilles Renaud, L'éclatement de la profession en service social (Montréal, éd. Coopératives Albert Saint-Martin, 1978), 62 et ss.

70. APETR, fonds Charles-Édouard-Bourgeois, boîte Service social, chemise Conseil canadien du Bien-être, M ${ }^{\mathrm{gr}}$ Alphonse Giroux, Les structures d'un conseil provincial de Bien-être, dact., 8 p. Il 
vite compris la nouvelle donne dans le secteur de l'assistance et du bienêtre au Québec, et ils cherchent à y jouer un rôle.

Car une telle prolifération d'organismes témoigne bien, en elle-même, de la stature que prend désormais l’État québécois dans le domaine de l'assistance et du bien-être. C'est parce que leurs membres québécois ne se reconnaissent pas assez dans le Conseil canadien de bien-être social et dans l'Association canadienne des travailleurs sociaux qu'ils créent des organismes proprement québécois, mieux en mesure de se faire entendre auprès des décideurs gouvernementaux. La Fédération des services sociaux à la famille et le Conseil du bien-être du Québec se donnent d'ailleurs explicitement une vocation de planisme, comme on dit à l'époque, de coordination, d'orientation à l'échelle du Québec et ils voudraient bien influencer les politiques de l'État providence ${ }^{71}$. Ces deux organismes et quelques autres, du reste, sont directement issus de CaritasCanada, où ils ont commencé à exister sous la forme de commission régulière ou de comité d'étude ${ }^{72}$.

Or, en 1964, la Conférence des évêques catholiques du Canada demande à Caritas-Canada de changer radicalement de cap. Les évêques l'avaient créée afin «de promouvoir la coordination des activités d'assistance, de charité et de bien-être; de constituer un centre d'information, d'études et de recherche; et de représenter les organisations de charité catholiques auprès des pouvoirs publics ${ }^{73}{ }^{\prime}$; sous l'influence notamment de l'évolution doctrinale encouragée par Vatican II $^{74}$, ils exigent maintenant qu'elle se métamorphose en un centre de réflexion sur la pastorale caritative et de diffusion de la pensée chrétienne sur la charité. En fait, alors que les forces

s’agit d'un document de consultation rédigé par le comité chargé d'évaluer la possibilité de mettre sur pied un tel conseil. Voir aussi dans le même dossier : Lettre de Louis Beaupré, secrétaire du Conseil de bien-être du Québec, à $M^{\mathrm{gr}}$ Charles-Édouard Bourgeois, président du CSS du diocèse de Trois-Rivières, Valleyfield, 9 décembre 1964.

71. CM, 110.18, Lettres patentes du Conseil de Bien-être du Québec, dact., 4 p. Le Conseil de bienêtre du Québec mettra toutefois beaucoup de temps à se concrétiser, comme en font foi les nombreux documents conservés dans le fonds Charles-Édouard-Bourgeois aux APETR. Voir aussi Louis Beaupré, «Politiques sociales au Québec», loc. cit.

72. Caritas-Canada (section française), Rapport annuel 1964 (Montréal, Secrétariat national, 1964), dact., 6. Parmi les finalités à l'origine de Caritas-Canada, il y avait aussi la participation aux activités de Caritas-Internationalis, une organisation du Vatican pour l'aide internationale.

73. Ibid.

74. Au concile Vatican II, l'Église a reconnu les droits humains, y compris les droits des gens à une vie décente. Elle a ainsi assigné l'aide aux pauvres et aux démunis non plus à la charité, mais en premier lieu à la justice, et donc à la responsabilité de l'État. Cette évolution doctrinale explique en partie que l'Église québécoise ait dans bien des cas encouragé les congrégations religieuses à se départir de leurs établissements de bien-être social en faveur de l'État. 
de l'Église déclinent partout en Occident - et au Québec à une vitesse désormais vertigineuse -, les évêques ont décidé de rassembler celles qui restent autour de projets proprement ecclésiastiques et religieux. Du coup, comme en cascade, éclosent un peu partout les projets de restructuration des conseils d'œuvres diocésains, de manière à définir plus précisément les rôles spécifiques des Fédérations d'œuvres et des Caritas proprement dites $^{75}$. Commence alors à Trois-Rivières une période d'assez grande incertitude. Alors qu'en 1961 le comité de la campagne de la Fédération était devenu une simple commission de Caritas-Trois-Rivières ${ }^{76}$, accentuant ainsi l'unité d'action de l'organisme, c'est au contraire vers une plus nette séparation qu'on se dirige ensuite. L'importante Commission des œuvres disparaît en 1964, et avec elle un lieu de réunion, d'échange et de mises en commun des organismes régionaux de bien-être ${ }^{77}$. Durant toute l'année 1965, enfin, le conseil d'administration étudie les moyens de diviser Caritas-Trois-Rivières en deux unités très distinctes, l'une pour la collecte de fonds et leur distribution aux ouvres de charité, et l'autre, à vocation de plus en plus pastorale ${ }^{78}$.

Incertitudes et réorientations précipitent le déclin, qu’avait provoqué l'attrition des ressources financières à la disposition de Caritas-TroisRivières. Il devient de plus en plus difficile d'atteindre le quorum aux séances du conseil d'administration ${ }^{79}$. Par ailleurs, les travailleurs sociaux professionnels laissent un à un l'organisme. En 1962, Gilles Lacroix était déjà retourné travailler au Centre de service social; à partir de 1964, il est membre du nouveau Conseil supérieur de la Famille, dont vient de s'outiller le ministère de la Famille et du Bien-être social ${ }^{80}$. L'année suivante, Jean-Paul Ramsay accepte la direction du Service des personnes âgées du ministère de la Famille et du Bien-être social. Ce n'est que l'une des nombreuses personnes qui, durant ces années, quittent les organismes de l'Église diocésaine pour donner désormais leur expertise et leur expérience à l'État providence né en partie d'elle. En 1964, M ${ }^{\text {gr }}$ Bourgeois

75. Voir notamment A. Desilets et L. Brunelle-Lavoie, L'Action sociale à Sherbrooke, op. cit., chapitre 3. Voir aussi CM, 100A.0168, Cahier des procès-verbaux..., 21 avril 1965.

76. AETR, boîte 535, chemise 52-15-10, Règlement $n^{\circ} 3,30$ mai 1960. Ce règlement semble être entré en vigueur en janvier 1961.

77. AETR, boîte 535, chemise 52-15-10, Règlement $n^{\circ}$ 4, février 1964.

78. CM, 100A.0168, Cahier des procès-verbaux..., 10 janvier 1966.

79. Ibid., 14 octobre et 16 décembre 1964.

80. APETR, fonds Charles-Édouard-Bourgeois, boîte Service social, chemise Divers/1, Lettre de Jules Perron, directeur exécutif du CSS du diocèse de Trois-Rivières, à Richard Sarrazin, directeur exécutif de la Fédération des services sociaux à la famille, Trois-Rivières, 18 août 1965. 
délaisse à son tour la direction administrative de l'organisme; son remplaçant, Louis-Roland Paradis, ne fait qu'y passer, avant de rejoindre lui aussi, en 1965, l'équipe du Centre de service social. À Paradis lui-même succède non un travailleur social, mais plutôt un aide social diplômé, l'abbé Léo Girard, qui tente tant bien que mal de garder le fort ${ }^{81}$. En réalité, c'est le Centre de service social et non plus Caritas qui mène à partir de 1964 toutes les enquêtes de terrain: sur les ressources offertes aux mères célibataires dans le diocèse, sur les prisonniers, sur la délinquance, puis, en collaboration avec le Service d'urbanisme de Trois-Rivières, sur deux secteurs d'habitation du centre-ville considérés comme des taudis et dont on déplacera bientôt la population ${ }^{82}$. Après avoir perdu une partie de sa capacité de financement et presque tout son pouvoir d'orientation, Caritas est même vidée désormais de sa fonction d'étude et de recherche.

\section{CONCLUSION}

Caritas-Trois-Rivières cesse d'exister le 28 novembre $1966^{83}$. Elle est remplacée par deux nouveaux organismes indépendants l'un de l'autre, mais rattachés à l'évêque. La Commission diocésaine caritative "est partie intégrante de la pastorale d'ensemble par laquelle l'Église diocésaine, sous la gouverne de l'Évêque, des prêtres et religieux, réalise la mission qui lui est dévolue par le Christ ${ }^{84}{ }$; parmi ses membres fondateurs, plusieurs de ceux qu'on a rencontrés déjà au moment de la création du Conseil central des œuvres, en 1954, ces membres des élites traditionnelles de plus en plus traditionnelles. La Fédération des œuvres de charité, de son côté, réussit à attirer une nouvelle génération d'administrateurs, quoique issus du même monde: un agronome de Sainte-Geneviève de Batiscan, un avocat, le gérant de la caisse populaire de Louiseville, un autre d'un grand magasin de Trois-Rivières, et même, de nouveau, un concessionnaire d'automobiles! Avant sa disparition, en 1968, la plus grande réalisation

81. CM, 100A.0168, Cahier des procès-verbaux..., 6 février, 22 juin et 16 décembre 1964.

82. CM, 100A.0168, Cahier des procès-verbaux..., 6 février, 22 juin et 16 décembre 1964. APETR, fonds Charles-Édouard-Bourgeois, boîte Service social, chemise Conseil canadien du bien-être social, Lettre de Jules Perron, directeur exécutif du CSS du diocèse de Trois-Rivières, à Michael Wheeler, du CCBES, 23 mars 1967.

83. CM. 100A.0168, Cahier des procès-verbaux..., 28 novembre 1966.

84. CM, 100B.07, «Lettre circulaire de $\mathrm{M}^{\mathrm{gr}}$ Georges-Léon Pelletier, évêque de Trois-Rivières, aux prêtres du diocèse de Trois-Rivières, 18 juin 1968 ", in Cahier des procès-verbaux des réunions de la Commission diocésaine caritative, du 18 juin 1966 au 26 septembre 1968, non paginé. On s'attendrait à retrouver les procès-verbaux de la commission diocésaine caritative à l'évêché plutôt qu'à Centraide Mauricie. 
de la nouvelle Fédération des œuvres sera l'ouverture officielle d'un comptoir de charité à Trois-Rivières, "Chez Monsieur Vincent», où l'on vend à bas prix meubles et vêtements usagés. L'époque des centaines et des milliers de solliciteurs étant révolue, la Fédération a aussi le temps d'organiser le système de retenue à la source, déjà en vigueur dans plusieurs villes du Québec, par lequel la contribution des donateurs, effectuée par versements périodiques, est déduite directement de leur salaire ${ }^{85}$. Malgré tout, les recettes restent modestes, 143500 \$ environ en 1966, 128600 \$ l'année suivante ${ }^{86}$. Tandis que la Société Saint-Vincent-de-Paul se retire finalement de la Fédération, $\mathrm{M}^{\mathrm{gr}}$ Bourgeois est bien placé, en tant que délégué de l'Ordinaire, pour veiller à ce que le Centre de service social, "qui doit à tout prix rester un service privé ${ }^{87}$ », reçoive une grosse part des sommes recueillies.

Finalement, en 1968, la Commission diocésaine caritative est abolie et la Fédération des œuvres est absorbée dans le nouveau Conseil régional de bien-être de la Mauricie, fondé par un groupe de laïcs, surtout des hommes d'affaires, tout à fait détachés de l'évêché. La première campagne de charité est d'ailleurs mise sous la présidence d'un membre du club Kiwanis, si heureux de dire tout autour de lui que le Conseil n'a plus rien à voir avec l'évêque, que plusieurs paroisses, prêtres et congrégations religieuses refusent désormais de contribuer: on n'atteint même plus $100000 \$$ de dons à partir de $1968^{88}$. Lorsqu'elles se présentent devant le comité des budgets, les œuvres membres prennent bien la peine de dire que si le Conseil ne peut leur venir en aide, "elles peuvent obtenir facilement la différence du ministère de la Santé ${ }^{89}$ ", ou de celui du Bien-être social. Même le Service social de la Mauricie, dont $\mathrm{M}^{\mathrm{gr}}$ Bourgeois quitte la présidence en 1971, ne reçoit plus rien cette année-là en fonds privés. Pour un temps, les Trifluviens, comme les autres contribuables du Québec, ne veulent plus financer la bienfaisance privée. Au début des

85. AETR, boîte 535, chemise 52-15-9, Procès-verbal de la Première assemblée générale annuelle de la Fédération des ouvres de charité du diocèse de Trois-Rivières, dact., 10 p.

86. CM, 100.0269. Premier rapport annuel du Conseil régional de bien-être de la Mauricie, inc., 3 juin 1969.

87. CM, 100B.04C73, Cahier des procès-verbaux du comité des budgets, 31 mars 1969.

88. CM, 100.0269, Premier rapport annuel du Conseil régional de bien-être de la Mauricie, inc., 3 juin 1969. Aussi: CM, 100B.04C73, Cahier des procès-verbaux du comité des budgets, 10 mai 1971. La campagne rapporte 98749 \$ en 1968, 85995 \$ en 1969 et 92904 \$ en 1970, à une époque de grande inflation. Les chiffres montrent que la contribution du clergé, des congrégations religieuses et des paroisses diminue beaucoup, celle des industries aussi, tandis que s'effondre le soutien des corporations municipales.

89. CM, 100B.04C73, Cahier des procès-verbaux du comité des budgets, 7 avril 1969. 
années 1970, toujours, le Conseil du bien-être du Québec et la Fédération des Services sociaux à la famille s'éteignent à leur tour graduellement; même le Conseil supérieur de la famille, un organisme gouvernemental celui-là, mais qui avait beaucoup recruté parmi les experts issus des agences sociales privées, ne fait plus le poids face à la Direction de la planification sociale et de la recherche du ministère de la Famille et du Bienêtre social ${ }^{90}$. On est entré dans l'ère du tout à l'État, symbolisée par l'adoption, en 1971, de la Loi sur les services de santé et les services sociaux, qui marque temporairement le triomphe de l'État providence.

Dans les années 1960, l'exemple de Caritas-Trois-Rivières le démontre, les organismes diocésains liés à l'assistance et au bien-être se sont véritablement dissous dans l'État providence après avoir contribué à sa naissance.

90. Louis Beaupré, «Politiques sociales au Québec», loc. cit. 


\section{TABLEAU IA}

Les résultats des campagnes de souscriptions publiques

(1954-1965) (en dollars) ${ }^{1}$

\begin{tabular}{|c|c|c|c|c|c|c|}
\hline \multicolumn{3}{|c|}{$\begin{array}{c}\text { Collecte à Trois-Rivières } \\
\text { seulement }\end{array}$} & \multicolumn{2}{|c|}{$\begin{array}{c}\text { Collecte dans les } \\
\text { principales villes du diocèse }\end{array}$} & \multicolumn{2}{|c|}{$\begin{array}{c}\text { Collecte dans } \\
\text { l'ensemble du diocèse }\end{array}$} \\
\hline 1954 & 1955 & 1956 & 1957 & 1958 & 1959 & 1960 \\
\hline 87637 & 89063 & 91285 & 97568,15 & 168538,38 & 186799,56 & 173713,50 \\
\hline \multicolumn{5}{|c|}{$\begin{array}{l}\text { Collecte dans l'ensemble du diocèse, mais } \\
\text { en même temps que la campagne diocésaine SPES }\end{array}$} & \multicolumn{2}{|c|}{$\begin{array}{l}\text { Collecte dans l'ensemble } \\
\text { du diocèse }\end{array}$} \\
\hline 1961 & & 1962 & & 1963 & 1964 & 1965 \\
\hline 61842,90 & Entr & 142000 & $145000^{2}$ & 142803 & 151512,66 & 152698,80 \\
\hline
\end{tabular}

TABLEAU IB

Les résultats des campagnes de souscriptions publiques (1966-1970) (en dollars) ${ }^{3}$

\begin{tabular}{ccccc}
$\begin{array}{c}\text { Collecte dans l'ensemble } \\
\text { du diocèse effectuée par la nouvelle } \\
\text { Fédération des œuvres }\end{array}$ & $\begin{array}{c}\text { Collecte dans l'ensemble du diocèse effectuée } \\
\text { par le nouveau Conseil régional de } \\
\text { bien-être de la Mauricie }\end{array}$ \\
\hline 1966 & 1967 & 1968 & 1969 & 1970 \\
143478,79 & 128594,59 & 98749 & 85896 & 92904,23 \\
\hline
\end{tabular}

1. Les références exactes concernant chacun des montants mentionnés ici sont données dans les notes accompagnant le texte de l'article. La campagne de 1965 est la dernière qu'organise Caritas-Trois-Rivières.

2. CM, 100A.0168, Cahier des procès-verbaux des réunions du conseil d'administration de Caritas-Trois-Rivières, du 17 septembre 1959 au 28 novembre 1966, 185-325: «On a recueilli 114000 \$; on peut espérer 142 à 145000 \$》. Le montant définitif n’a pas été retrouvé dans les sources consultées.

3. Même remarque sur les références que pour le tableau 1a. Ce tableau rassemble les informations données en conclusion de l'article. La campagne de 1970 est la dernière avant l'adoption de la Loi sur les services de santé et les services sociaux. 